Editorial

\title{
Inventions 2017 Best Paper Award
}

\author{
Inventions Editorial Office
}

MDPI AG, St. Alban-Anlage 66, 4052 Basel, Switzerland; inventions@mdpi.com

Received: 23 August 2017; Accepted: 23 August 2017; Published: 24 August 2017

Inventions is instituting an annual award to recognize the outstanding papers published in the journal.

We are pleased to announce the first "Inventions Best Paper Award" for 2017. Nominations, chosen from all papers published in 2016, were made by the Editorial Board. Following review by the Editorial Board, the following three top-voted research articles, in no particular order, have won "Inventions Best Paper Awards" for 2017:

Yuenyong Nilsiam and Joshua M. Pearce

Open Source Database and Website to Provide Free and Open Access to Inactive U.S. Patents in the Public Domain

Inventions 2016, 1(4), 24; doi:10.3390/inventions1040024

Available online: http:/ /www.mdpi.com/2411-5134/1/4/24

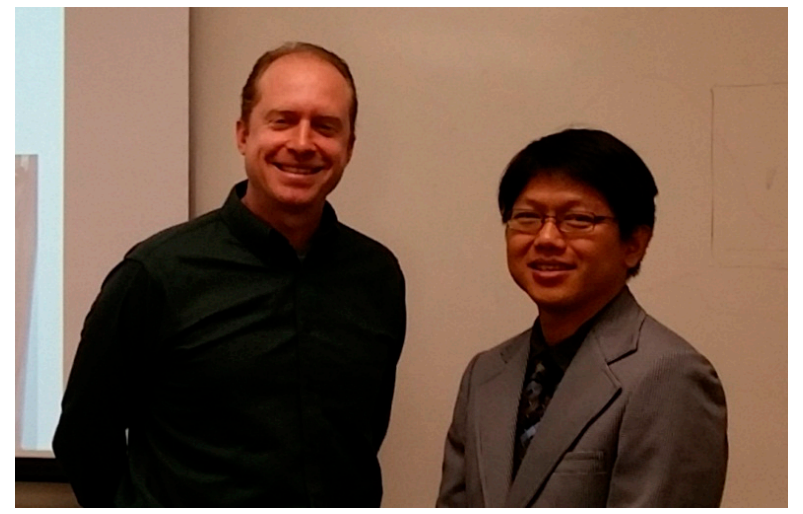

Yuenyong Nilsiam (right) and Joshua M. Pearce (left).

A new patent search tool developed by Dr. Yuenyong Nilsiam (now at King Mongkut's University of Technology North Bangkok, Thailand) and Professor Joshua Pearce at Michigan Technological University in the USA makes it easier to discover and track inactive patents.

Many innovators and inventors feel they have squandered hours fruitlessly rifling through old patents on the US Patent and Trademark Office website, trying to figure out which are still active and how they may relate to developing technologies.

The team streamlined the exploration process with an online search engine, which is now free for everyone to use at freeip.mtu.edu. They tested how well the tool works, which was published in Inventions.

With today's fast-paced innovation, 20 years is a long time to wait-and it is how long a patent's registration lasts. However, nearly half of all registered patents are inactive before their lifetime ends, which is a potential goldmine for inventors and innovators. So, the algorithm works by scraping the U.S. Patent Database each week when the agency updates patent statuses. Every 3.5, 7.5 and 11.5 years, 
patents come up for renewal, and if the dues are not paid and the paperwork remains unfiled, then the patent becomes inactive and enters the public domain.

"We want to make patents useful again to inventors," Pearce says, adding the search tool is part of a larger movement. "More companies and individuals are looking to open source hardware development, which allows you to harness a global ecosystem of innovators, engineers and designs."

Pearce runs the Michigan Tech Open Sustainability Technology (MOST) Lab and is deeply committed to making knowledge widely available. The team hopes that by initially streamlining inactive patent searches, it will foster and speed up innovation down the road-particularly in the rapidly developing world of open source hardware.

Fotis D. Kanellos, Amjad Anvari-Moghaddam and Josep M. Guerrero

Smart Shipboard Power System Operation and Management Inventions 2016, 1(4), 22; doi:10.3390/inventions1040022

Available online: http:/ /www.mdpi.com/2411-5134/1/4/22

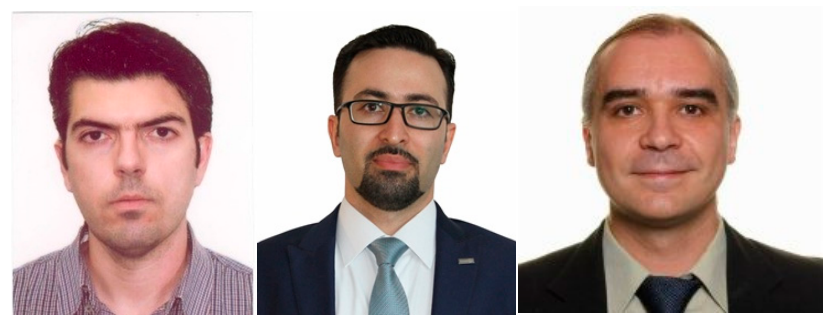

Fotis D. Kanellos, Amjad Anvari-Moghaddam and Josep M. Guerrero (from left to right).

It is expected that, in 2025, yearly transport by sea will increase three times in comparison with 2004. At the same time, there is a worldwide growing concern regarding the efficient operation of ships and the greenhouse gas (GHG) emissions produced by them, which has led to stricter regulations for their environmental impact and operation efficiency.

The extensive electrification of ship power systems, widely known as All-Electric Ship (AES), has been proved a very promising solution for the improvement of ship efficiency and limitation of GHG emissions. In AES, electric power is produced by large generating sets which supply both ship electric loads and large electric motors used for ship propulsion. AES technology provides operation flexibility as a large variety of power plant components can be exploited. However, the ship power system becomes more complex and challenging power management problems emerge. The most significant is to design a sophisticated power management system that will optimally plan the operation of the on-board power plants and electric loads. Well-planned operation of a shipboard electrical system at the supply and demand side, in particular the electric propulsion demand, can greatly affect its overall efficiency.

In this paper, a practical framework for optimal operation scheduling in AES with regard to operation cost minimization, GHG emissions limitation and several technical/environmental constraints is proposed. The problem is formulated as a mixed-integer nonlinear programming model and solved using a metaheuristic algorithm based on Particle Swarm Optimization. To demonstrate the applicability and superiority of the proposed method, several test scenarios based on an AES RO-PAX ferry are examined, and the obtained simulation results are analyzed and compared to those of conventional operation strategies.

\section{Sheng-Joue Young, Chia-Lin Chiou, Yi-Hsing Liu and Liang-Wen Ji}

Synthesis of Ga-Doped $\mathrm{ZnO}$ Nanorods by Hydrothermal Method and Their Application to Ultraviolet Photodetector

Inventions 2016, 1(1), 3; doi:10.3390/inventions1010003

Available online: http:/ /www.mdpi.com/2411-5134/1/1/3 


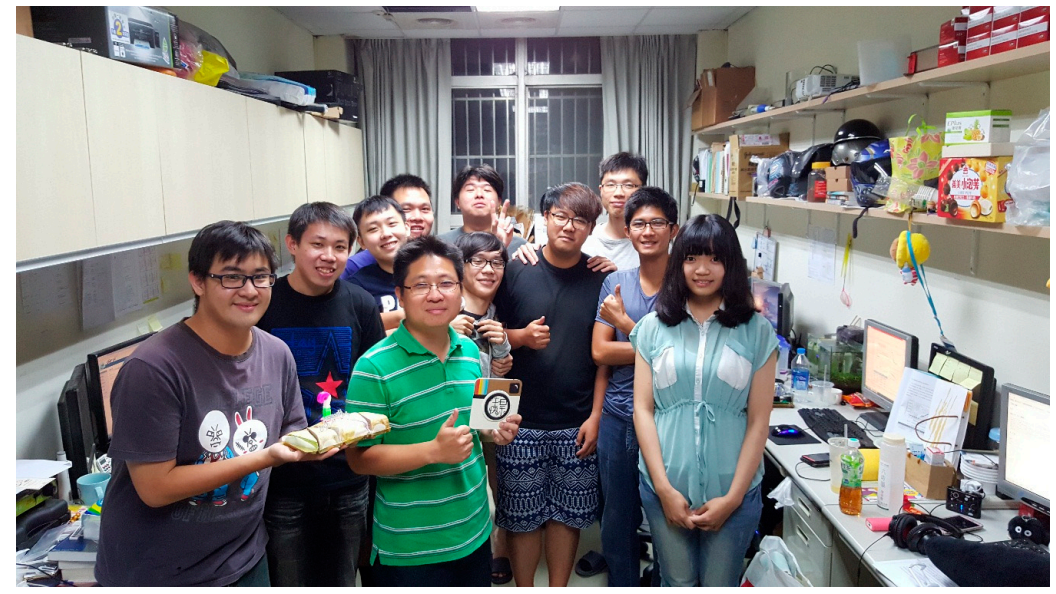

Sheng-Joue Young and his team.

Researchers have recently investigated zinc oxide $(\mathrm{ZnO})$ nanostructures because of their unique properties and promising applications, including in optical, electronic, and light-emitting devices, ultraviolet (UV) photodetectors (PDs), and solar cells. The characteristics of ZnO include a wide band-gap energy $(3.37 \mathrm{eV})$, large exciton binding energy $(60 \mathrm{meV})$, and easy growth on various substrates. Researchers have concentrated on the growth and application of these nanostructures, including nanorods, nanowires, and nanodots, using various methods, including the vapor-liquid-solid, chemical vapor deposition, and thermal evaporation methods. However, vapor methods require complex procedures, sophisticated equipment, or rigid experimental conditions.

In this study, high-density single crystalline Ga-doped $\mathrm{ZnO}$ (GZO) nanorods were grown on glass substrate by the hydrothermal method. The structural and optoelectronic properties of Ga-doped $\mathrm{ZnO}$ nanorods were studied. The microstructure of the GZO was studied by scanning electrical microscope. The structural characteristics of the GZO were measured by X-ray diffraction. It was found that the peaks related to the wurtzite structure $\mathrm{ZnO}$ (100), (002), and (101) diffraction peaks. The (002) peak indicates that the nanorods were preferentially oriented in the c-axis direction. The existence of $\mathrm{Ga}$ was examined by energy diffraction spectra, indicating that the $\mathrm{Ga}$ atom entered into the $\mathrm{ZnO}$ lattice. The optical properties of the GZO were measured by photoluminescence spectra. It was found that all GZO nanorod arrays showed two different emissions, including ultraviolet and green emissions. GZO nanorod metal-semiconductor-metal UV PDs were also fabricated. The photo-current and dark-current constant ratio of the fabricated PDs was approximately 15.2 when biased at $1 \mathrm{~V}$.

We believe that these three exceptional papers are valuable contributions to Inventions and the scientific research field. On behalf of the Inventions Editorial Board, we would like to congratulate these teams for their excellent work. A certificate will be given to each of them.

We would like to take this opportunity to thank all the nominated research groups of the above exceptional papers for their contributions to the Inventions, and thank the Inventions Editorial Board for voting and helping with this "Best Paper Award".

The Editorial Board and Editorial Staff at Inventions is committed to meeting the needs of the scientific community by providing useful and timely reviews of all manuscripts submitted, and providing an open access journal for your results. Please consider submitting your work to Inventions, and we look forward to announcing your paper as an Inventions Best Paper in the future.

Prize Awarding Committee

Inventions Editorial Board

(C) 2017 by the author. Licensee MDPI, Basel, Switzerland. This article is an open access article distributed under the terms and conditions of the Creative Commons Attribution (CC BY) license (http:/ / creativecommons.org/licenses/by/4.0/). 